\title{
PH棌ENIX
}

PX No. 208

BNL No. 69030

\section{Calorimetry for Global Measurements in PHENIX}

Sebastian N. White for the PHENIX Collaboration

Brookhaven National Laboratory, Upton, NY, USA

Published in Nucl. Phys. A698 (2002) 420c-423c

Physics Department

Brookhaven National Laboratory Operated by

Brookhaven Science Associates

Upton, NY 11973

Under Contract with the United States Department of Energy

Contract Number DE-AC02-98CH10886 


\title{
Calorimetry for Global Measurements in PHENIX
}

\author{
Sebastian N. White ${ }^{\mathrm{a}}$ for the PHENIX collaboration * \\ ${ }^{a}$ Brookhaven National Lab, Upton, N.Y 11973
}

\section{Introduction}

The charged particle multiplicity, $\frac{d N_{c h}}{d \eta}$, and transverse energy, $E_{t}$, are fundamental aspects of Heavy Ion collisions which PHENIX has measured in Au-Au collisions at a centerof-mass (c.m.s.) energy $\sqrt{s_{N} N}=130 \mathrm{GeV} . E_{t}$ is a multiparticle global observable related, through the Bjorken relation, to the maximum energy density achieved in the collision. For a given c.m.s. energy, the multiplicity and transverse energy are expected to increase with the number of nucleons participating in a collision $\left(n_{\text {part }}\right)$. The dependence on $n_{\text {part }}$ is found to be $\left(\frac{d E_{t}}{d \eta}, \frac{d N_{c h}}{d \eta}\right) \sim n_{\text {part }}^{\alpha}$, where $\alpha$ is larger than unity. This rapid growth (i.e. more rapid than linear) is not suprising in the collision of large nuclei since an individual participant nucleon may undergo more than one collision.

The variable $n_{\text {collision }}$ is also introduced to denote the number of binary collisions. Whereas $n_{\text {part }}$ can be directly measured, in principle, directly from the number of "nonparticipating" nucleons detected in the forward direction, $n_{\text {collision }}$ is not observable but can be calculated statistically from $n_{\text {part }}$, using the Glauber model.

The Zero Degree Calorimeters (ZDC's) were built to measure $n_{\text {part }}$ with the limitation that at a collider we can only observe the free neutron(and less well the proton) component(s) of the "non-participating" nucleons. The derivation of $n_{\text {collision }}$ from these data is treated by Denisov in these proceedings[3].

What is our expectation for $\frac{d E_{t}}{d \eta}$ and its variation with c.m.s. energy and multiplicity? There were a number of calculations presented by Eskola and others.

Let's assume, for simplicity, that particles are produced, on average, with the same mean transverse momentum $\left\langle p_{t}\right\rangle$, no matter what the multiplicity. In this case we would predict a growth with c.m.s. energy (or multiplicity) of: $\left(\left.\frac{d E_{t}}{d \eta}\right|_{\eta=0}\right)=<E_{t}^{\text {part }}>\cdot \rho^{\text {tot }}(0)$, where $\rho^{c h}\left(\eta_{0}\right)=\left.\frac{d N_{c h}}{d \eta}\right|_{\eta=\eta_{0}}$ and we take $\rho^{t o t} \simeq \frac{3}{2} \cdot \rho^{c h}$, which would be exact, due to isospin conservation, if all produced particles were pions. The c.m.s. energy dependence of $\rho^{\text {ch }}(0)$ is well measured for elementary $\mathrm{N}-\mathrm{N}$ collisions (mostly $\bar{p} p[7]$ ), so we trivially estimate a $50 \%$ growth of $\left(\left.\frac{d E_{t}}{d \eta}\right|_{\eta=0}\right)$ at RHIC compared with SPS, in good agreement with what we measure[6].

The search for structure in the dependence of $\left\langle p_{t}\right\rangle$ on $\rho$ has a long history and it is instructive to see what happens at fixed energy. Recently it was argued[4] that a

${ }^{*}$ For the full PHENIX collaboration author list and acknowledgements see, contribution by W.A.Zajc in this volume. 
monotonic dependence according to $\left\langle p_{t}^{2}\right\rangle \propto \rho$ is seen in $\bar{p} p$ collisions[5] and should apply at RHIC. PHENIX data shows that this relation does not hold at RHIC, on average, but it would be interesting to study the dependence when $n_{\text {collision }}$ is held constant.

This discussion illustrates the central role of $E_{t}$ and $n_{\text {part }}$, which PHENIX measures with calorimeters. Details of the analyses may be found elsewhere[6],[3]. In what follows we focus on the instrumentation and its performance.

\section{Measurement of $n_{\text {spectator }}$}

Small aperture two-arm hadron calorimeters (ZDC's) for the very forward region were built by our group for all of the RHIC Heavy Ion experiments. They were located $18 \mathrm{~m}$ downstream of the interaction point, after a $3.5 \mathrm{~T}$ accelerator dipole magnet, which sweeps essentially all charged particles out of the $(2 \mathrm{mrad})$ aperture seen by the ZDC's. A dedicated test at CERN[2] and, more recently, PHENIX data[3] demonstated that the ZDC energy $\left(\propto n_{\text {spectator }}^{\text {neutron }}\right)$ measures $n_{\text {part }}$ except in the most peripheral collisions.

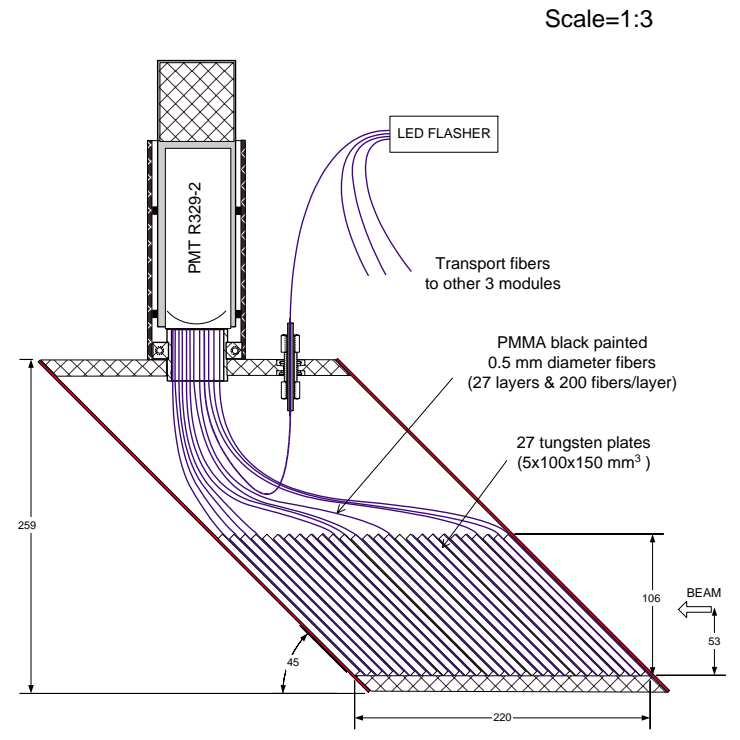

Figure 1. A ZDC module.

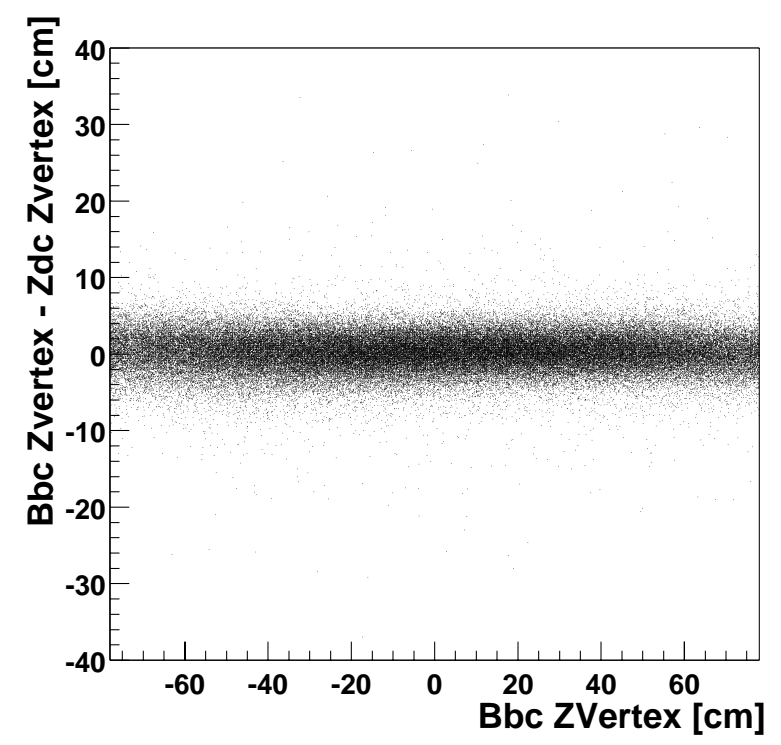

Figure 2. ZDC vs.Bbc vertex reconstruction $(\sigma=2.3 \mathrm{cms})$.

\section{ZDC technology}

Each ZDC consists of 3 independently read out modules, one behind the other, of $2 \cdot \lambda_{\text {Interaction }}$ depth. There are 2 basic requirements which led to our ZDC design:

- the transverse dimension of the ZDC's is limited to $\leq 10 \mathrm{~cm}$ (i.e. only $\sim 1$. $\left.\lambda_{\text {Interaction }}(W)\right)$ by the maximum possible separation between the 2 RHIC beamtubes at their location.

- The ZDC is used to measure the interaction position along the beam $\left(z_{\text {vertex }}\right)$ and the event "start" time by comparing time-of-flight(TOF) to the 2 arms, complementing 
similar measurements by the Beam-Beam Counter hodoscope(Bbc's). This requires a timing precision of $\sim 100-150$ ps.

Both requirements are well suited to the use of a dense absorber(tungsten) interleaved with ribbons of PMMA fiber (with index $\mathrm{n}=1.49$ and numerical aperture n.a.=0.5). The fiber ribbons generate and capture Cerenkov light produced by fast, forward-going particles in hadronic showers and therefore are primarily sensitive to the shower core. Better than $92 \%$ response uniformity, for hadrons impacting within $\pm 4 \mathrm{~cm}$ (i.e. to within $1 \mathrm{~cm}$ of the detector edge), and an energy resolution of $\frac{\sigma_{E}}{E}=19 \% @ 100 \mathrm{GeV}$ was demonstrated in the CERN test beam[1]. A single ZDC module is shown in Fig. 1. The timing performance was studied by comparing the ZDC and Bbc vertex measurements and is illustrated in Fig. 2, where we find $\sigma\left(z_{\text {vertex }}^{B b c}-z_{\text {vertex }}^{Z D C}\right)=2.3 \mathrm{~cm}$, which implies better than 100 ps for both detectors. Because of the large separation between the ZDC's, they were an invaluable tool for analyzing accelerator performance and backgrounds throughout the interaction regions in the early RHIC stores, wherein $\sigma_{\text {interaction }}$ was $\simeq 70 \mathrm{~cm}$.

\section{Central EM Calorimeter}

The PHENIX $\mathrm{Pb} /$ Scint calorimeters detect particles near mid-rapidity $(|\eta|<0.35)$ at a radial distance of $5.2 \mathrm{~m}$ from the interactions. The calorimeter consists of 15,552 nonprojective towers and is finely segmented $\left(\Delta_{\eta}, \Delta_{\phi} \simeq 0.011\right)$ in order to keep the tower occupancy below $\sim 5 \%$ in central Au-Au collisions. A 4 tower module consists of 66 alternating $\mathrm{Pb}\left(0.25 X_{0}\right)$ and scintillator (5mm polystyrene $+0.01 \% P O P O P+2.5 \%$ p-terphenyl). The design emphasizes EM shower resolution (high sampling frequency) and speed (use of fast fluors). The wave shifter fiber fluor (Bicron BCF99-29a) was adopted from $R \& D$ for the SDC EM calorimeter. The $1 \mathrm{~mm}$ diameter waveshifter fibers penetrate the module and pass through $1.3 \mathrm{~mm}$ holes which are formed in the injection molding of the scintillators("shashlik" readout).

Gain and timing stabilization relies on the use of a fiber-optic light distribution network and a single Nd-Yag laser- frequency tripled to the UV (355 nm)- which simultaneaously excites all $(15,552 \cdot 66)$ scintillators in the calorimeter. The network is designed to generate the time ditribution and depth profile of $1 \mathrm{GeV}$ electron showers.

The PHENIX EM calorimeter development extended over several years with emphasis on optimizing speed, uniformity and light yield as well as an "industrial approach" to the production of a high performance, fine grained calorimeter covering $\sim 50 \mathrm{~m}^{2}$.

\section{Measurement of $E_{t}$}

The calorimeter was designed for high resolution measurement of EM showers initiated by electrons and photons (primarily from $\pi^{0}$ decay). However, because of the small average kinetic energy and high multiplicity of produced particles in a typical heavy ion collision, both the $E_{t}$ energy scale and fluctuations can be computed reliably from the detected energy, assuming that the calorimeter response to EM showers was calibrated properly.

Modules were exposed to a test beam at the Brookhaven AGS and their response to electrons as well as muons and a variety of hadrons was measured. For calibration purposes, the signal from muons either traversing the calorimeter transversely (similar to 
the main flux of cosmic rays in the normal orientation of the calorimeter) or longitudinally (similar to the high flux of non-interacting $\pi^{ \pm}$collected in PHENIX data) was compared to that of $1 \mathrm{GeV}$ electrons. Cosmic rays and MIP particles were used to precalibrate the calorimeter and adjust individual channel gains. The final calibration was obtained with identified electrons in PHENIX, and confirmed by our reconstructed $\pi^{0}$ mass $[9]$. The energy scale uncertainty is $\simeq \pm 1.5 \%$.

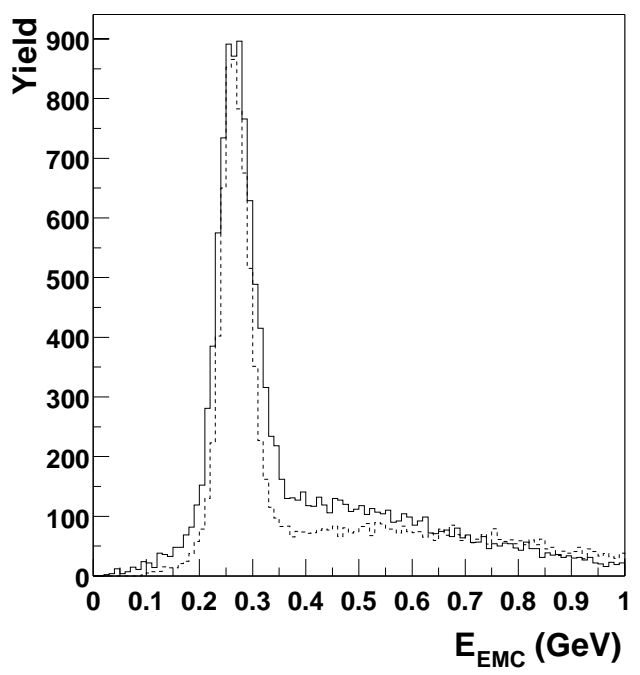

Figure 3. Lineshape for $1 \mathrm{GeV}$ testbeam identified $\pi^{ \pm}$-(dashed) and PHENIX tracks.

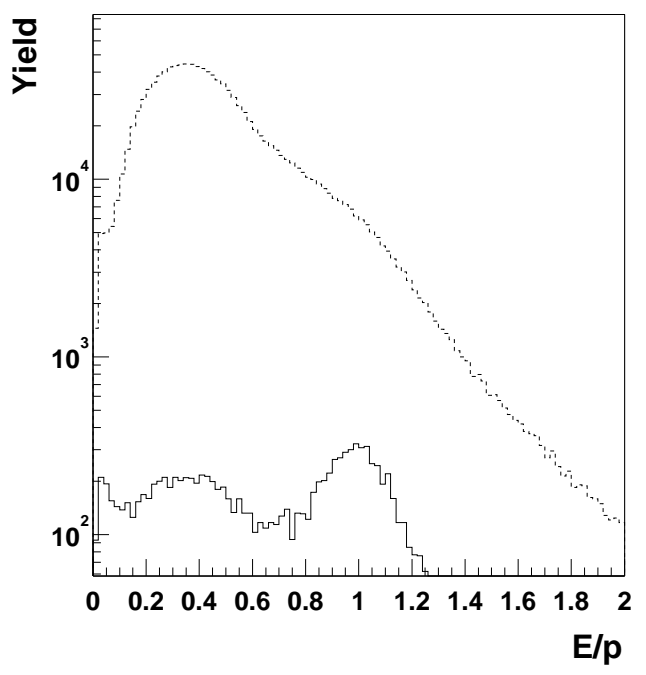

Figure 4. $\quad E^{c a l} /$ momentum of raw tracks (upper curve) and RICHidentified electrons..

\section{REFERENCES}

1. Details on construction and performance of the ZDC's may be found in C.Adler et al., nucl-ex/0008005.

2. H. Appelshauser et al. [NA49 Collaboration], Eur. Phys. J. A2, 383 (1998).

3. A.Denisov, these Proceedings.

4. L. McLerran and R. Venugopalan,Phys. Rev. D 49, 2233 (1994).

5. Alexopoulos et al.,Phys. Rev. D 48,984 (1993).

6. A.Milov, these Proceedings.

7. "Proton-Antiproton Collider Physics",G.Altarelli and L.diLella eds., World Scientific (1989).

8. see S. White, Nucl. Instrum. Meth. A360, 212 (1995) or follow www.phenix.bnl.gov/phenix/WWW/emcal/papertrail/papers.html

9. G.David, these proceedings. 